\title{
Ab initio simulations of the surface free energy of $\mathrm{TiN}(001)$
}

\author{
Axel Forslund $\odot^{*}$ \\ KTH Royal Institute of Technology, SE-100 44 Stockholm, Sweden \\ Xi Zhang $\odot$ and Blazej Grabowski \\ Institute of Materials Science, University of Stuttgart, Pfaffenwaldring 55, D-70569 Stuttgart, Germany \\ Alexander V. Shapeev \\ Skolkovo Institute of Science and Technology, Skolkovo Innovation Center, Nobel Street 3, Moscow 143026, Russia \\ Andrei V. Ruban $\odot$ \\ KTH Royal Institute of Technology, SE-100 44 Stockholm, Sweden \\ and Materials Center Leoben Forschung GmbH, A-8700 Leoben, Austria
}

(Received 23 February 2021; revised 30 April 2021; accepted 10 May 2021; published 20 May 2021)

\begin{abstract}
The temperature dependence of the surface free energy of the industrially important $\operatorname{TiN}(001)$ system has been investigated by means of an extended two-stage upsampled thermodynamic integration using Langevin dynamics (TU-TILD) methodology, to include the fully anharmonic vibrational contribution, as obtained from $a b$ initio molecular dynamics (AIMD). Inclusion of the fully anharmonic behavior is crucial, since the standard lowtemperature quasiharmonic approximation exhibits a severe divergence in the surface free energy due to a hightemperature dynamical instability. The anharmonic vibrations compensate for the quasiharmonic divergence and lead to a modest overall temperature effect on the TiN(001) surface free energy, changing it from around $78 \mathrm{meV}^{-2}$ at $0 \mathrm{~K}$ to $73 \mathrm{meV} \AA^{-2}$ at $3000 \mathrm{~K}$. The statistical convergence of the molecular dynamics is facilitated by the use of machine-learning potentials, specifically moment tensor potentials, fitted for $\mathrm{TiN}(001)$ at finite temperature. The surface free energy obtained directly from the fitted machine-learning potentials is close to that obtained from the full AIMD simulations.
\end{abstract}

DOI: 10.1103/PhysRevB.103.195428

\section{INTRODUCTION}

It has recently been recognized that anharmonicity can drastically impact defect formation, even at temperatures significantly below the melting point when there is a breakdown of local symmetry as, e.g., for atoms next to a vacancy $[1,2]$. In such cases, the quasiharmonic approximation (QHA), nowadays widely used in combination with ab initio calculations, cannot even qualitatively reproduce the temperature dependence of the defect formation free energy.

One may expect anharmonic effects to be pronounced for other geometrically open defects as well; for example, for surfaces where atoms in the outermost layer have the freedom to move much further out of the bulk than into the bulk. Such a behavior was indeed observed in Monte Carlo calculations of the (001) surface of $\mathrm{Al}$ using a classical interatomic

\footnotetext{
*axefor@kth.se
}

Published by the American Physical Society under the terms of the Creative Commons Attribution 4.0 International license. Further distribution of this work must maintain attribution to the author(s) and the published article's title, journal citation, and DOI. Funded by Bibsam. potential [3]. The surface free energy in fully anharmonic calculations strongly deviated from the results obtained with the QHA.

Nevertheless, so far there has only been one ab initio surface study including the impact of anharmonicity [4]. In Ref. [4], two approaches, producing similar results, were applied to the $\mathrm{TiO}_{2}(110)$ surface: thermodynamic integration of the (1) internal energy over temperature and (2) stress over strain while simultaneously splitting the initial bulk supercell into slabs at a fixed temperature. The relation to the QHA was not investigated. Because such explicitly anharmonic calculations are in general computationally highly demanding, all other $a b$ initio surface studies, of which there are in any case only very few so far, were performed within the QHA [5-9].

The availability of an efficient $a b$ initio approach to compute anharmonic surface free energies would be beneficial from several perspectives. From an applied point of view, it would enable access to accurate surface properties for industrially important high-temperature materials such as, e.g., coatings for abrasive wear resistance, precipitates in steels or cemented carbides, or thin films used in electronics or optics. From a more fundamental perspective, accurate hightemperature surface energies are the only way to get a reliable connection to experimental data since surface energies of solids are measured at temperatures close to the melting point 
[10]. Therefore, the main aim of this paper is to devise an $a b$ initio methodology, based on density-functional theory (DFT), for efficient and accurate calculations of the surface free energy with total account of anharmonic effects, so that the remaining error can be ascribed to the used approximation for the exchange-correlation functional in the DFT approach.

For that purpose, we extend the previously developed twostage upsampled thermodynamic integration using Langevin dynamics (TU-TILD) method [2,11] to surface free-energy calculations. The TU-TILD method has already been used for free-energy calculations of various systems, including vacancy formation free energies [2] and stacking fault free energies [12]. Combined with machine-learning potentials, the computational demands can be substantially decreased [13] by reducing the number of expensive DFT calculations.

Here, we apply the extended TU-TILD method to determine the temperature dependence of the surface free energy of $\mathrm{TiN}(001)$. TiN is widely used as a coating material in different applications due to its wear resistance [14]. Although there exist several $a b$ initio calculations of the (001) surface [15], which is likely the most stable one at $0 \mathrm{~K}$ for TiN, nothing is known about its temperature dependence. TiN has the $\mathrm{NaCl}$-structure (B1), exhibits mainly covalent bonding with a metallic type density of states and has a quite high Debye temperature of $900 \mathrm{~K}[16,17]$. The temperature dependence of bulk properties in TiN has been investigated before. The studied bulk properties include the heat capacity [18-20] and the thermal expansion coefficient $[18,19,21]$ within the QHA with a focus on lower temperatures, and elastic properties [22] from $a b$ initio molecular dynamics (AIMD).

\section{METHODOLOGY}

\section{A. Surface free energy}

We use the slab technique to determine the surface free energy $\gamma(T)$ according to

$$
\gamma(T)=\frac{F_{\text {slab }}\left(a_{T}, T\right)-F_{\text {bulk }}\left(a_{T}, T\right)}{2 A_{T}},
$$

where $F_{\text {slab }}\left(a_{T}, T\right)$ and $F_{\text {bulk }}\left(a_{T}, T\right)$ refer to the Helmholtz free energies of the slab and bulk with the same number of atoms, for the lattice constant $a_{T}$ and temperature $T$, and $A_{T}$ is the surface area of the slab. The factor of $\frac{1}{2}$ accounts for the two, alike surfaces in the slab calculation. The lattice constant $a_{T}$ corresponds to the equilibrium one in the bulk $a_{\mathrm{eq}}(T)$ at the given temperature and at zero pressure. For the slab geometry $a_{T}$ fixes the two, lateral dimensions within the surface plane. Since the additional degree of freedom perpendicular to the surface plane is unconstrained and a homogeneous lattice distortion of the slab in this direction is possible, it is assumed here that the lateral interatomic distances in the surface planes are the same as in the corresponding layers in the infinite bulk. The interplane distances can be different for several surface layers, while they converge to their bulk value in the middle of the slab with increasing thickness. [As discussed below, eight layers provide converged results in the specific case of $\operatorname{TiN}(001)$.]

The Helmholtz free energies (here we omit the slab and bulk subscripts) can be adiabatically decomposed into the following contributions:

$$
F\left(a_{T}, T\right)=E\left(a_{T}\right)+F^{\mathrm{el}}\left(a_{T}, T\right)+F^{\mathrm{vib}}\left(a_{T}, T\right),
$$

where $E$ denotes the conventional $0 \mathrm{~K}$ total energy of the system (either bulk or slab), $F^{\mathrm{el}}$ the thermal-electronic-excitation contribution for the static lattice, and $F^{\mathrm{vib}}$ the vibrational free energy of the lattice, obtained here either in the QHA or in the fully anharmonic form using the TU-TILD method. When calculated with the latter method, the vibrational free energy $F^{\mathrm{vib}}$ contains the (adiabatic) coupling between one-electron excitations and lattice vibrations.

To determine an accurate thermal expansion at zero pressure required to fix $a_{T}$, the full free-energy surface $F_{\text {bulk }}(V, T)$ as a function of volume $V$ and temperature $T$ is calculated for the bulk including the same contributions as above,

$$
F_{\text {bulk }}(V, T)=E_{\text {bulk }}(V)+F_{\text {bulk }}^{\mathrm{el}}(V, T)+F_{\text {bulk }}^{\text {vib }}(V, T) .
$$

The thermal expansion is determined through the minima with respect to the volume on this surface. In our implementation, we perform a Legendre transformation of $F_{\text {bulk }}(V, T)$ to the Gibbs energy surface $G_{\text {bulk }}(P, T)$, where $P$ is the pressure, from which the equilibrium volume is obtained through the derivative with respect to pressure. Other equilibrium thermodynamic properties of the bulk, e.g., the heat capacity at constant pressure, are accessible from $G_{\text {bulk }}(P, T)$ as well. As already stated, the surface free energy is given at zero pressure, and all the results for the bulk are also given for zero pressure. The difference to ambient pressure bulk results is negligible.

\section{B. Anharmonic free energy calculations and machine learning potentials}

To calculate the full vibrational free energy including the anharmonic contribution, a modified version of the original TU-TILD method [2,11] is used:

$$
F^{\mathrm{vib}}=F^{\text {Einst }}+F^{\text {Einst } \rightarrow \mathrm{MTP}}+F^{\mathrm{MTP} \rightarrow \mathrm{DFT}},
$$

where

$$
\begin{gathered}
F^{\text {Einst } \rightarrow \text { MTP }}=\int_{0}^{1} \mathrm{~d} \lambda_{1}\left\langle E^{\mathrm{MTP}}-E^{\text {Einst }}\right\rangle_{\lambda_{1}}, \\
F^{\mathrm{MTP} \rightarrow \mathrm{DFT}}=\int_{0}^{1} \mathrm{~d} \lambda_{2}\left\langle E_{\mathrm{low}}^{\mathrm{DFT}}-E^{\mathrm{MTP}}\right\rangle_{\lambda_{2}}+\langle\Delta E\rangle^{\mathrm{UP}} .
\end{gathered}
$$

Further, $F^{\text {Einst }}$ is the free energy of an optimized Einstein crystal; $E^{\text {Einst }}, E^{\mathrm{MTP}}$, and $E_{\text {low }}^{\mathrm{DFT}}$ are the energies of a particular atomic configuration calculated for the Einstein crystal, with a moment tensor potential (MTP) [23] as implemented in the MLIP software [24], and with low-converged DFT parameters, respectively; $\langle\ldots\rangle_{\lambda}$ denotes a thermodynamic average for a particular coupling constant $\lambda$ and at a certain temperature and volume/lattice constant; finally, the term $\langle\Delta E\rangle^{\mathrm{UP}}$ is obtained within free-energy perturbation theory and accounts for the difference in free energy between the low- and highconverged DFT calculations [11].

The first modification with respect to the original TU-TILD method is the usage of an optimized Einstein crystal as the analytic reference to compute the absolute free energy, instead of a quasiharmonic reference. The reason for abandoning 
the quasiharmonic reference is the occurrence of imaginary frequencies at larger lattice constants for the present material system (cf. discussion in Sec. IV), as well as computational efficiency for large and complicated structures. The Einstein crystal is a simple and convenient workaround [25]. The corresponding Einstein frequency can be chosen quite arbitrarily within a reasonable interval specific for this system. Since the Einstein system is used only as an auxiliary reference for thermodynamic integration, the choice does not affect the final result obtained after the integration. However, we note that a careful study of the supercell size dependence is important for this integration, as discussed in Sec. III A. Further, for the corresponding slab calculations, the choice of the reference positions around which the Einstein crystal is calculated is important as discussed in Sec. III B.

The second modification is the usage of a machine-learning potential, specifically MTP, as a highly efficient bridge between the analytical reference system and the DFT system. Very recently, MTPs have been proven to be efficient in combination with the TU-TILD method [13]. In summary, MTPs describe the atomic environment of the $i$ th atom by the moments of inertia of the neighboring atoms $j$,

$$
M_{\mu, \nu}=\sum_{j} f_{\mu, i, j}\left(r_{i j}\right) \underbrace{\mathbf{r}_{i j} \otimes \cdots \otimes \mathbf{r}_{i j}}_{v \text { times }},
$$

where $f_{\mu, i, j}\left(r_{i j}\right), \mu=1,2, \ldots$ are radial functions and define different shells around the $i$ th atom, and $\mathbf{r}_{i j} \otimes \cdots \otimes \mathbf{r}_{i j}$ is a $v$-dimensional tensor. Contractions of these moments into a scalar then serve as basis functions, whose linear combination, with parameters fitted to data, can approximate the local interaction energy. (For more details, see Ref. [23].) The MTPs are also used here to directly calculate the anharmonic vibrational surface free energy. This provides a significant speed-up compared to running the computationally heavy AIMD, while still providing results in good approximation, as will be discussed in Sec IV B.

\section{COMPUTATIONAL DETAILS}

All DFT calculations were done using the PAW method [26] as implemented in the Vienna ab initio simulation package (VASP) [27,28]. We used the titanium PAW potential with the semi-core $p$-electrons included in the valence band $\left(\mathrm{Ti}_{\mathrm{pv}}\right)$ and the nitrogen PAW soft-core potential $\left(\mathrm{N}_{\mathrm{s}}\right)$, as provided in VASP version 5.4.4. The generalized gradient approximation (GGA) in the PBE parametrization [29] was used for the exchange correlation energy.

\section{A. Bulk free energy calculations}

The bulk free-energy surface $F_{\text {bulk }}(V, T)$ was obtained by computing the different free-energy contributions according to Eq. (3). In general, the convergence parameters were chosen such as to achieve an accuracy in the free energy of below $1 \mathrm{meV} /$ atom.

The $0 \mathrm{~K}$ energy $E_{\text {bulk }}(V)$ was computed for a 32-atom supercell built from a $1 \times 1 \times 4$ expansion of the conventional cubic unit cell for consistency with the slab calculations, as described in Sec. III B. It was computed on a mesh of six volume points in the relevant range, as required to cover the thermal expansion. The plane-wave cutoff was set to $500 \mathrm{eV}$ and the $k$-point mesh to $17 \times 17 \times 5$. The Methfessel-Paxton scheme [30] of order 1 was utilized with a smearing width of $0.1 \mathrm{eV}$. The computed energy points were fitted to the Vinet equation of state [31].

The electronic free energy $F_{\text {bulk }}^{\text {el }}(T, V)$ for the static lattice was computed for the primitive unit cell (two atoms) using the self-consistent field approach [32] within finitetemperature DFT [33]. We used a mesh of 13 volume times 14 temperature points (up to $3000 \mathrm{~K}$ ). The plane-wave cutoff was set to $500 \mathrm{eV}$ and the $k$-point mesh to $25 \times 25 \times 25$. The temperature-volume dependence was parametrized as discussed in Ref. [34]. We used in particular a fourth order fit for the temperature expansion of the density of states and a third order polynomial for the volume dependence of the free energy.

The vibrational free energy $F_{\text {bulk }}^{\text {vib }}(V, T)$ was computed according to the prescription in Eq. (4). An MTP was trained to energies and forces from in total 9000 atomic configurations obtained from initial DFT AIMD runs for the bulk at $3000 \mathrm{~K}$ and a set of six volumes. The other specifications for the bulk initial DFT AIMD were adjusted to the ones for the low-converged DFT runs in the TU-TILD method (discussed below). In particular, a 128-atom supercell built from a $4 \times 4 \times 4$ expansion of the primitive unit cell (two atoms), a plane-wave cutoff of $350 \mathrm{eV}$, and a $2 \times 2 \times 2$ Monkhorst-Pack [35] $k$-point mesh were used. The MTP had a cutoff radius of $4.5 \AA$, including the three nearest-neighbor shells. The level of the MTP was chosen to be 20. The level is a degree-like hyperparameter controlling the number of MTP parameters [24], which in the present work was equal to 410. The resulting root-mean-square error (RMSE) of the energy difference between DFT and MTP was $2 \mathrm{meV} /$ atom and the RMSE of the force was $0.16 \mathrm{eV} \AA^{-2}$.

The difference in free energy between the Einstein crystal and the MTP, $F^{\text {Einst } \rightarrow \text { MTP }}$, was calculated in a 512 -atom supercell built from a $4 \times 4 \times 4$ expansion of the conventional cubic unit cell (eight atoms). A large enough supercell is important for this integration to capture the contribution of long wavelength phonons to the free energy. With the chosen 512-atom supercell the corresponding free-energy contribution is converged to below $1 \mathrm{meV} /$ atom even for the highest temperature and largest lattice constants (Fig. 1). A dense mesh of 10 volume times 12 temperature points was chosen for the calculation of $F^{\text {Einst } \rightarrow \text { MTP }}$. At each volume-temperature point, a dense set of $\lambda_{1}$ values (typically 26) was used for the integration in Eq. (5). At each $\lambda_{1}$ value statistically very well converged results were obtained from long MD simulations (50000 steps). Note that for the computation of $F^{\text {Einst } \rightarrow \text { MTP }}$ no expensive DFT runs are required.

The difference in free energy between the MTP and DFT, $F^{\mathrm{MTP} \rightarrow \text { DFT }}$, was obtained in a 128 -atom supercell build from a $4 \times 4 \times 4$ expansion of the primitive unit cell (two atoms). Note that this free energy difference converges much more rapidly with supercell size than $F^{\text {Einst } \rightarrow \text { MTP }}$. Test calculations show that $F^{\mathrm{MTP} \rightarrow \text { DFT }}$ changes by less than $2 \mathrm{meV} /$ atom between a 128- and 512-atom supercell. A mesh of three volume times five temperature points was chosen for the calculation of $F^{\mathrm{MTP} \rightarrow \mathrm{DFT}}$ (corresponding lattice constants $(\AA)$ : 4.2, 4.3, 


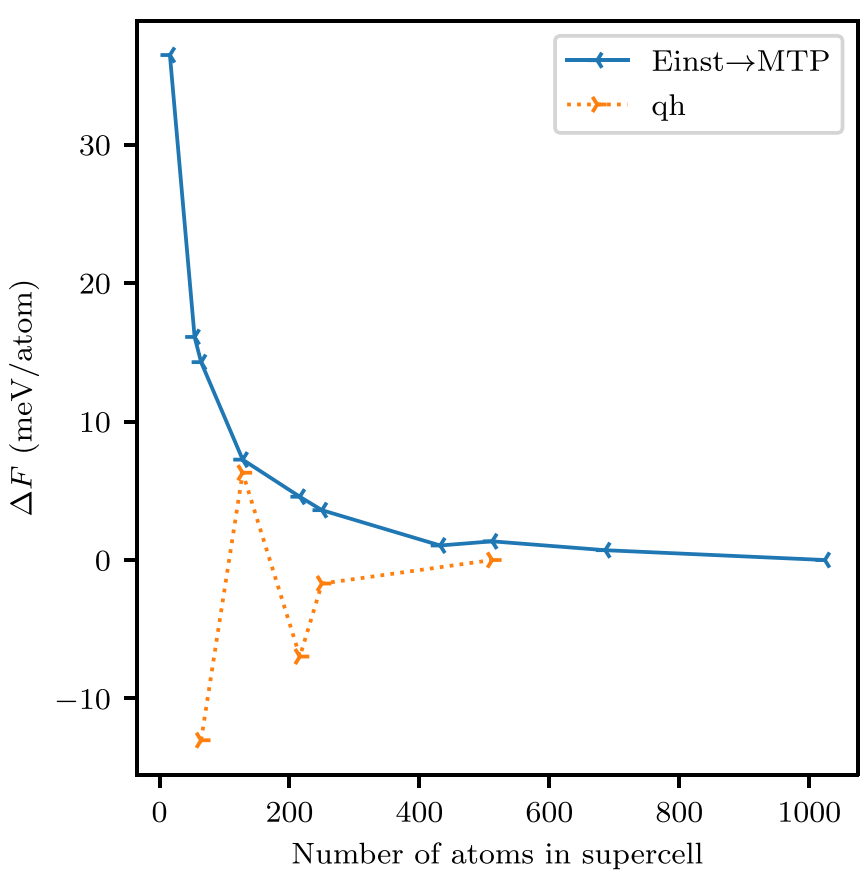

FIG. 1. Convergence of the bulk free energies with respect to the supercell size at $3000 \mathrm{~K}$ and at a lattice constant of $4.4 \AA$. The results for $F^{\text {Einst } \rightarrow \text { MTP }}$ are shown by a blue line, and $F^{\text {qh }}$ by a dotted orange line. The difference in free energy $\Delta F$ is given with reference to the last value in each set.

4.4; temperatures (K): 250, 500, 1000, 2000, 3000). At each volume-temperature point, five $\lambda_{2}$ values $(0,0.25,0.5,0.75$, 1) were chosen for the integration in Eq. (6). At each $\lambda_{2}$ value, two MD runs initiated with different random seeds were conducted to achieve simulation lengths of about 1000 MD steps (excluding equilibration). This sampling resulted in statistical errors of below $0.2 \mathrm{meV} /$ atom, due to the excellent performance of the MTP in predicting the DFT energetics (cf. also Sec. IV). For the low-converged DFT parameters $\left[E_{\text {low }}^{\mathrm{DFT}}\right.$ in Eq. (6)], we used a plane-wave cutoff of $350 \mathrm{eV}$ and a $2 \times 2 \times 2$ Monkhorst-Pack [35] $k$-point mesh. For the highconverged parameters [entering the last, upsampling term in Eq. (6)], we used $500 \mathrm{eV}$ and a $6 \times 6 \times 6 k$-point mesh, and about 15 upsampling calculations at each volume-temperature point, which was more than enough to obtain well converged energies. The impact of lattice vibrations on the electronic free energy was included both in the corresponding AIMD and at the stage of the upsampling. The explicitly computed mesh of volume-temperature points for $F^{\mathrm{MTP} \rightarrow \mathrm{DFT}}$ was parametrized with a polynomial expansion in order to obtain an analytical description of $F^{\mathrm{MTP} \rightarrow \mathrm{DFT}}(V, T)$ as function of $V$ and $T$. Specifically, we used a polynomial up to third order in both $\mathrm{V}$ and $\mathrm{T}$.

The two vibrational contributions $F^{\text {Einst } \rightarrow \text { MTP }}$ and $F^{\mathrm{MTP} \rightarrow \text { DFT }}$ were added together on the dense mesh used to calculate the former of the contributions. Additionally, the quantum-mechanical Einstein free energy $F^{\text {einst }}$ was added on top, such as to obtain the final vibrational free energy $F_{\text {bulk }}^{\text {vib }}$ [as specified in Eq. (4)] on the dense set of volume-temperature points. These points were then parametrized utilizing the

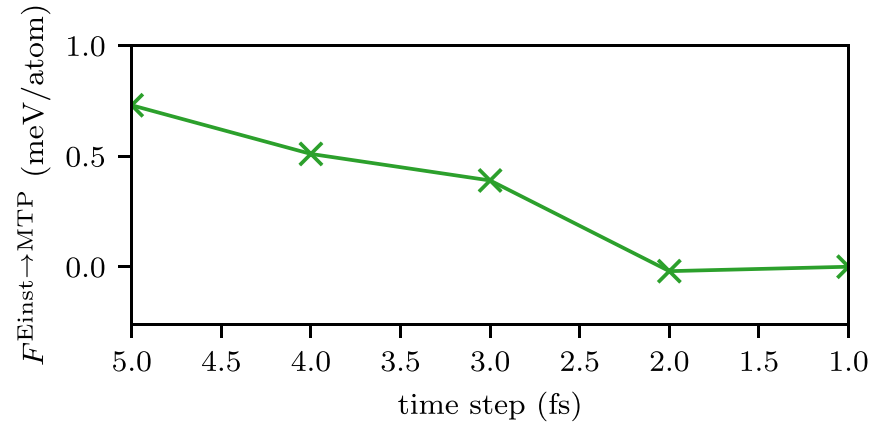

FIG. 2. Convergence of the time step in the integration from an Einstein crystal reference to the bulk-MTP for $4.4 \AA$ and $3000 \mathrm{~K}$.

analytical formula

$$
\begin{aligned}
F_{\text {bulk }}^{\mathrm{vib}}(V, T)= & \frac{\hbar}{2} \omega(V) \\
& +\sum_{i}^{n} C_{i} k_{B} T \ln \left[1-\exp \left(-\frac{\hbar \omega_{i}(V)}{k_{B} T}\right)\right],
\end{aligned}
$$

where $\omega(V)$ is expanded in a polynomial in $V$ up to second order, $\omega_{i}(V)$ a polynomial in $V$ up to third order and $C_{i}$ is an additional fitting parameter. Using $n=3$, already gives good fitting results. The starting parameters for the fit were adjusted to the low-temperature QHA results. This fitting procedure follows in spirit the one introduced in Ref. [36] [Eq. (29)/(30) in that reference] where a renormalized anharmonic frequency was introduced to fit the anharmonic free energy. Here, we have extended the ansatz to account for the full vibrational free energy.

All the MD simulations were run with a Langevin thermostat [36] with a damping parameter of $0.01 \mathrm{fs}^{-1}$. The van-Gunsteren-Berendsen algorithm [37] was used for the integration of Newton's equations of motion in the time stepping. A 2 fs time step was deemed sufficient for all AIMD runs (cf. Fig. 2).

\section{B. Slab free energy calculations}

In principle, $F_{\text {slab }}\left(a_{T}, T\right)$ needs to be calculated only along the thermal expansion of the bulk $\left[a_{T}=a_{\mathrm{eq}}(T)\right]$. However, to increase numerical stability and to easily be able to account for changes in the thermal expansion (induced for example by accounting for thermal contributions from the electronic system), we extended the mesh of investigated lattice constants at each temperature. That is, we computed $F_{\text {slab }}(a, T)$ at several (lateral) lattice constants $a$ around the required equilibrium lattice constant $a_{T}$. A parametrization of $F_{\text {slab }}(a, T)$ was then used to obtain $F_{\text {slab }}\left(a_{T}, T\right)$.

All contributions to $F_{\text {slab }}(a, T)$ were calculated with eight atomic layers perpendicular to the surfaces of the slab and with a vacuum region of $4 a(\approx 17 \AA)$ inserted between the two surfaces. Figure 3 shows that the $0 \mathrm{~K}$ and (full) vibrational contributions are converged to within $1 \mathrm{meV}^{-2}$ for a slab with eight layers. Further computational details specific to the contributions to $F_{\text {slab }}(a, T)$ are provided in the following, however only if they differed from the respective bulk calculations; otherwise, we refer to the parameter settings given in Sec. III A. 
(a)

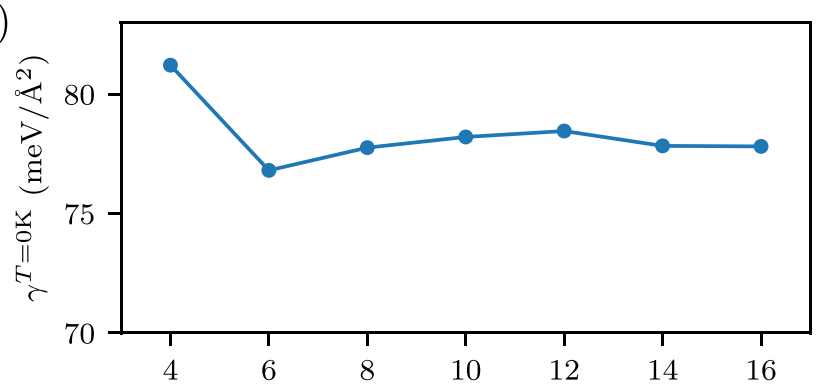

(b)

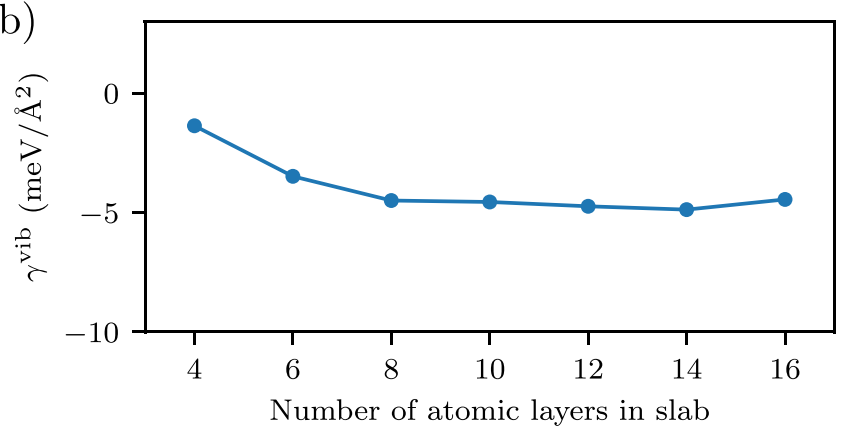

FIG. 3. Convergence of the (a) DFT surface energy at $0 \mathrm{~K} \gamma^{T=0 \mathrm{~K}}$ and (b) MTP vibrational surface free energy $\gamma^{\text {vib }}$ at $2000 \mathrm{~K}$ with respect to the number of atomic layers in the slab. The lattice constant is fixed close to $a_{T}$ for the corresponding temperature $(a=4.25 \AA$ for $0 \mathrm{~K}$ and $a=4.35 \AA$ for $2000 \mathrm{~K}$ ). The MTP corresponds to the one fitted to the slab calculations ("slab-MTP").

The $0 \mathrm{~K}$ energy $E_{\text {slab }}(a)$ was computed in a slab of 32 atoms built from a $1 \times 1 \times 4$ expansion of the conventional cubic unit cell with eight atoms (plus the vacuum region), at six different lattice constants $a$. At each lattice constant atomic relaxation was allowed perpendicular to the surface for two surface layers. A $k$-point mesh of $17 \times 17 \times 5$ was used. The difference $E_{\text {slab }}(a)-E_{\text {bulk }}(a)$ was parametrized with a third order polynomial and included together with the other contributions to the surface free energy.

The electronic contribution $F_{\text {slab }}^{\mathrm{el}}(a, T)$ was calculated in the same 32-atom slab supercell as $E_{\text {slab }}(a)$, on a mesh of six lattice constants times six temperatures. The $k$-point mesh was set to $17 \times 17 \times 5$. For consistency, the same fitting procedure was applied as in the case of $F_{\text {bulk }}^{\text {el }}(T, V)$, by utilizing an effective volume derived from the (lateral) lattice constant $a$.

For the calculation of $F_{\text {slab }}^{\mathrm{vib}}(a, T)$ a second, separate MTP ("slab-MTP") was fitted to optimize the TU-TILD slab calculations. In the same way as for the bulk-MTP, we utilized initial DFT AIMD runs for the slab at $3000 \mathrm{~K}$ and several lattice constants with the other specifications as for the low-converged runs (see below; specifically a 512-atom slab, $350 \mathrm{eV}$ cutoff, and the $\Gamma$ point). The level of the slab-MTP was the same as for the bulk-MTP.

The difference in free energy between the Einstein crystal and the slab-MTP, $F^{\text {Einst } \rightarrow \text { MTP }}$, was calculated in a 512-atom supercell built from a $4 \times 4 \times 4$ expansion of the conventional cubic unit cell with $4 a$ of vacuum along the $z$ direction. A mesh of six lattice constants times 12 temperature points was used for the calculation of $F^{\text {Einst } \rightarrow \text { MTP }}$. The other parameters were set as for the respective bulk calculations, except for the important difference in the choice of the reference positions around which the Einstein contribution was calculated. Due to the fact that the mean positions of the surface atoms are shifted outwards with respect to the $0 \mathrm{~K}$ relaxed positions (see the discussion in Sec. IV D), the reference positions for the Einstein crystal had to be adjusted to the mean position from the MD. Taking the $0 \mathrm{~K}$ relaxed positions instead gives a poor overlap of the phase space covered by the Einstein crystal and the one from the slab-MTP, and thus a very bad convergence of the integration in Eq. (5).

The difference in free energy between the slab-MTP and DFT $F^{\mathrm{MTP} \rightarrow \text { DFT }}$ was obtained in a 512-atom slab build from a $4 \times 4 \times 4$ expansion of the conventional cubic unit cell with $4 a$ of vacuum along the $z$ direction. A total of nine lattice constant-temperature points were used for the explicit calculation of $F^{\mathrm{MTP} \rightarrow \mathrm{DFT}}$ around $a_{T}$. The same set of $\lambda_{2}$ values $(0,0.25,0.5,0.75,1)$ and different random seeds were used as for the respective bulk calculations. At each $\lambda_{2}$ value shorter simulation lengths (about 500 MD steps, excluding equilibration) were sufficient to achieve the same statistics due to the larger supercell in the slab than in the bulk calculations. For the low-converged DFT parameters, we used a plane-wave cutoff of $350 \mathrm{eV}$ and the $\Gamma$ point for sampling the Brillouin zone. For the high-converged parameters, we used $500 \mathrm{eV}$ and a $4 \times 4 \times 4 k$-point mesh. The explicitly computed $F^{\mathrm{MTP} \rightarrow \mathrm{DFT}}(a, T)$ points were parameterized in the same manner as for the respective bulk contribution by utilizing an effective volume derived from the (lateral) lattice constant $a$.

Finally, all the parametrized contributions were extracted at $a_{T}$ in the full temperature range and the surface free energy was calculated including all relevant excitation mechanisms according to Eq. (1).

\section{Quasiharmonic calculations}

The QHA was obtained with phonopy [38] using finite displacements of $0.015 \AA$ in the corresponding VASP calculations. The bulk phonons were sampled with a 250 -atom supercell based on a $5 \times 5 \times 5$ expansion of the primitive unit cell with two atoms. A plane-wave cutoff of $500 \mathrm{eV}$ and a $5 \times 5 \times 5 k$-point mesh were used for calculations at six different volumes. The volume dependence of the phonon free energies was fitted to a third order polynomial for each temperature up to $3000 \mathrm{~K}$ in steps of $1 \mathrm{~K}$.

In the case of the slab, we used the same 512-atom supercell as in the MD, a plane-wave cutoff of $500 \mathrm{eV}$ and a $3 \times 3 \times 1 k$-point mesh. The DFT dynamical matrix was obtained for a completely relaxed slab supercell, for six different volumes. However, we also used the slab-MTP to obtain dynamical matrices expanded around three different geometries (cf. Sec. IV B): (1) for a completely relaxed slab; (2) a slab with positions rescaled from the relaxed ones at $a_{\mathrm{eq}}(0 \mathrm{~K})$; and (3) a slab using mean positions from the slab-MTP MD. The forces from the slab-MTP were in good agreement with forces from DFT and gave the same qualitative behavior in the phonon free energy for the relaxed slab, as well as for a control calculation for the rescaled positions (at $a_{T}=4.25 \AA$ ).

The phonon free energy $F^{\mathrm{vib}}\left(a_{T}, T\right)$ was added to the electronic total energy $E\left(a_{T}\right)$ for the bulk as well as for the slab. The surface free energy $\gamma(T)$ within the QHA was then 


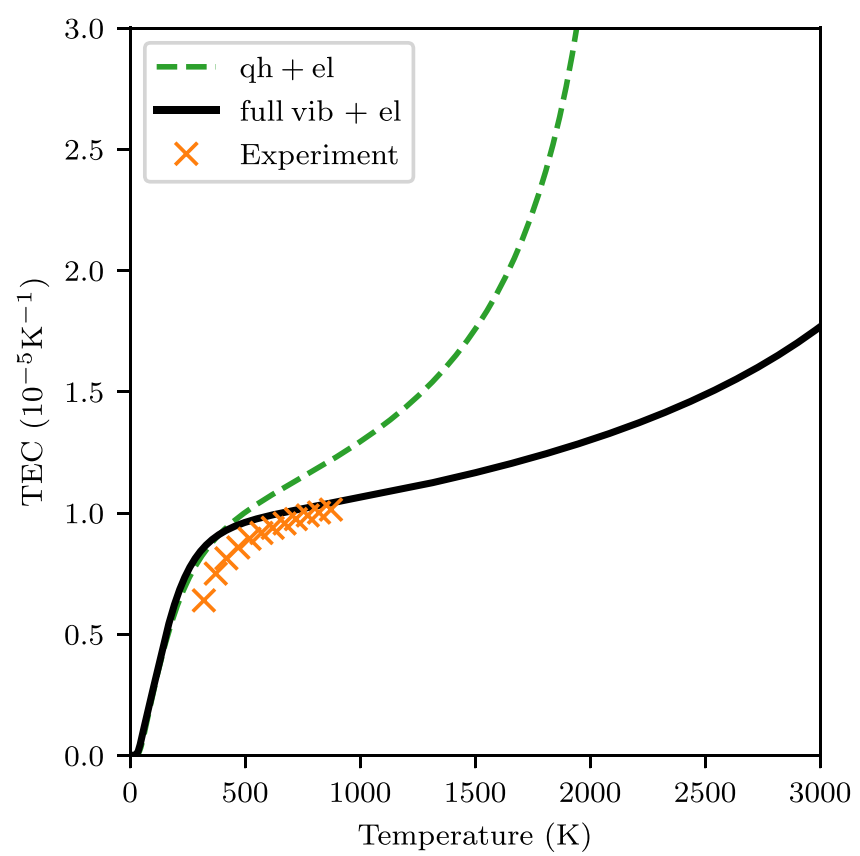

FIG. 4. Linear thermal expansion coefficient (TEC) for bulk TiN obtained in the QHA (dashed green line) and from AIMD including the full vibrational and electronic impact (black solid line). Experimental values (orange crosses) are from Ref. [21].

calculated according to Eq. (1). It should be noted, that for the different approximations that have been mentioned above (using relaxed, rescaled, and mean positions), $E\left(a_{T}\right)$ can differ substantially.

\section{RESULTS AND DISCUSSION}

\section{A. Bulk thermal properties of TiN}

In Fig. 4, the DFT (GGA-PBE) calculated thermal expansion coefficient (TEC) of TiN is shown together with experimental data [21]. The QHA breaks down at elevated temperatures and the corresponding TEC (green dashed) diverges well below the experimental melting point of $3220 \mathrm{~K}$ [39]. A similar diverging behavior of the QHA was reported earlier for $\mathrm{ZrC}$ (for GGA-PBE) by Duff et al. [11]. The breakdown of the QHA in the present TiN system is due to a dynamical instability that occurs at increased lattice constants. Analysis of the phonon spectrum reveals a softening of the longitudinal acoustic phonon mode for $q \approx 0.7 \frac{\pi}{a}(110)$, which eventually leads to imaginary frequencies at larger volumes. We note that the use of the PBEsol [40] functional decreases the thermal expansion; however, the dynamical instability persists at similar volumes.

Inclusion of explicit anharmonicity is essential to obtain accurate bulk properties for TiN at elevated temperatures. The full DFT curve including all relevant excitation mechanisms (black solid line in Fig. 4) is in good agreement with experimental data and it shows no diverging behavior. The experimental values for the TEC close to room temperature in fact exhibit larger uncertainty [21], which may explain the small discrepancy with DFT. The quality of the DFT results can be even better assessed for the heat capacity shown in Fig. 5, for which a wider range of experimental data is

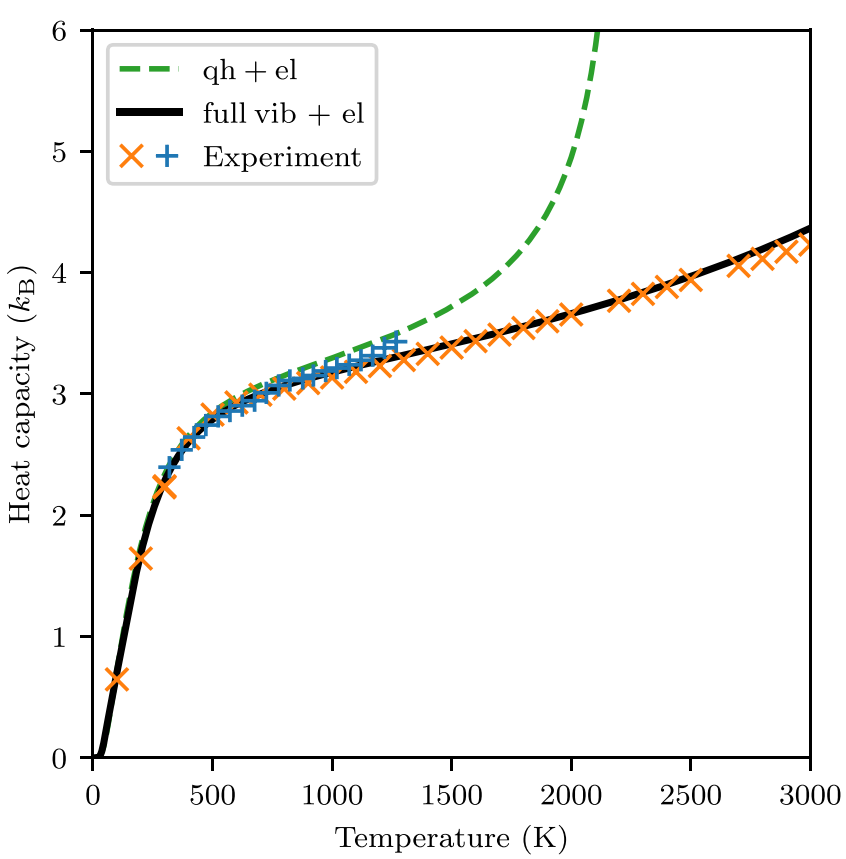

FIG. 5. Isobaric heat capacity for bulk TiN obtained in the QHA (dashed green line) and from AIMD including the full vibrational and electronic impact (black solid line). Experimental values are from Ref. [41] (orange crosses) and from Ref. [42] (blue pluses).

available. The heat capacity is, similarly as the expansion coefficient, a sensitive measure of the curvature of the freeenergy surface. Indeed, we observe an excellent agreement with experiments in the whole temperature range for the full DFT calculation including anharmonicity.

\section{B. Surface free energy of $\mathrm{TiN}(001)$}

Figure 6 shows the temperature dependence of the final DFT (GGA-PBE) surface free energy including the full, anharmonic vibrational contribution and electronic excitations (black solid line). There are no experimental data available and the present DFT data therefore serve as the first highaccuracy prediction of the surface energetics of $\mathrm{TiN}(001)$. Confidence in the DFT results was established in the last subsection based on the available experiments for bulk properties of TiN.

Overall we observe a temperature-induced decrease in the surface free energy of $-5 \mathrm{meV}^{2}\left(-0.08 \mathrm{~J} \mathrm{~m}^{2}\right)$, from $78 \mathrm{meV}^{-2}$ at $0 \mathrm{~K}$ to $73 \mathrm{meV} \AA^{-2}$ at $3000 \mathrm{~K}$. Thermal electronic excitations can be seen to have a noticeable impact (difference between orange solid and black solid line) at higher temperatures; they decrease the surface free energy by up to $3 \mathrm{meV}^{-2}$. The impact of zero-point vibrations is small. They decrease the surface free energy (at $0 \mathrm{~K}$ ) by $-1 \mathrm{meV} \AA^{2}\left(-0.08 \mathrm{~J} \mathrm{~m}^{2}\right)$. However, there is a compensation effect in play, because the zero-point contribution also increases the equilibrium $0 \mathrm{~K}$ lattice constant and this leads to a positive shift of the surface free energy by the same amount, such that the combined impact of the zero-point contribution is negligible.

The overall decrease in the full surface free energy of $\mathrm{TiN}(001)$ amounts to $-6 \%$ with reference to the absolute 


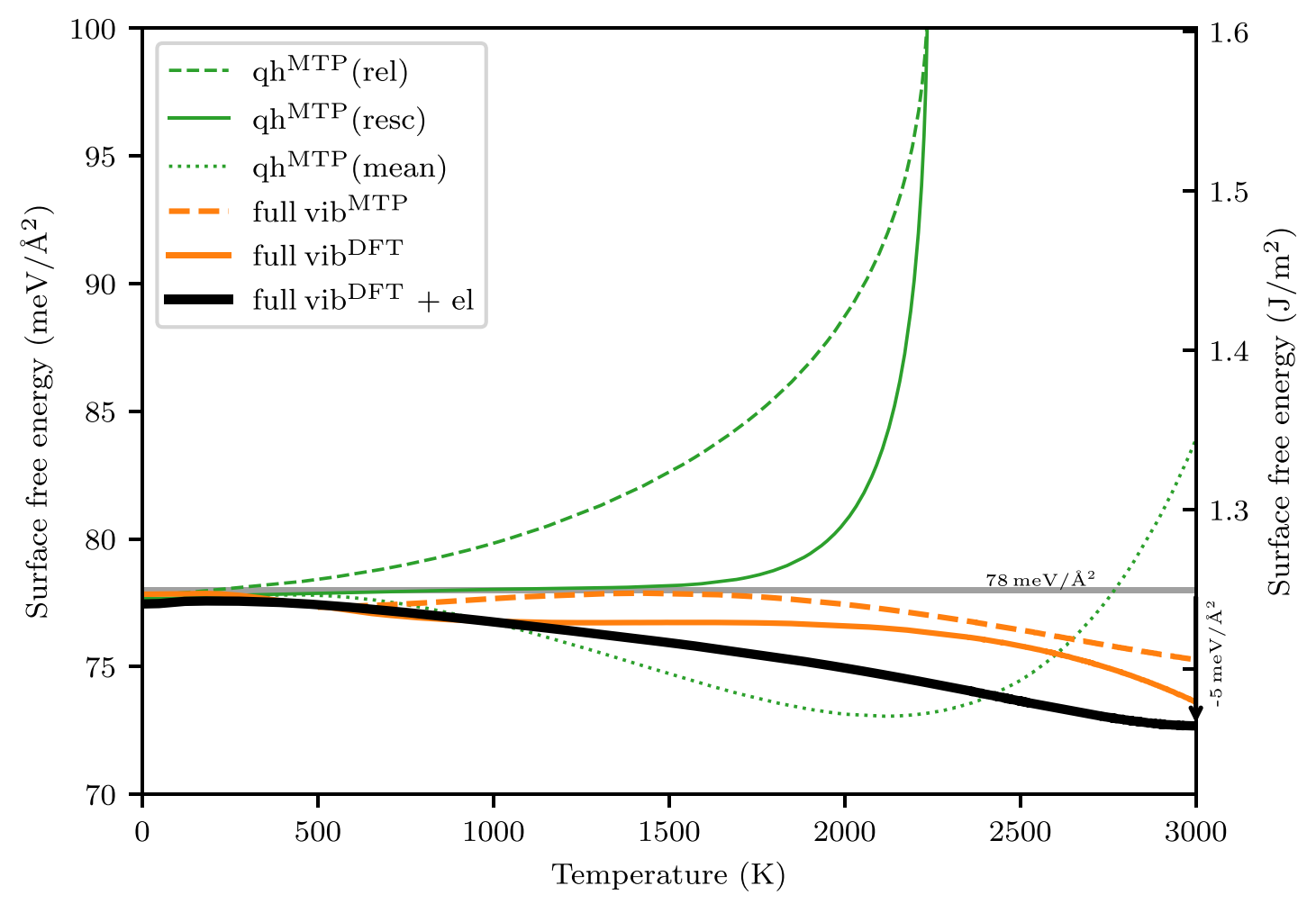

FIG. 6. Temperature dependence of the TiN(001) surface free energy predicted by fully anharmonic DFT calculations including the impact of electronic excitations (black solid line). The orange lines exclude the electronic excitations and show the full vibrational contribution obtained with DFT (solid orange) and from MTPs (dashed orange). The green lines represent the QHA results for the MTPs with different choices for the atomic positions at which the dynamical matrix is computed (see text for details). DFT QHA results are similar to the MTP results.

value of $78 \mathrm{meV}^{-2}$ at $0 \mathrm{~K}$. This relative change is rather small when compared to temperature-induced changes found from high-accuracy finite temperature DFT calculations for formation free energies of other defects. For example, for vacancy formation free energies, changes of about $-20 \%$ up to the melting temperature were observed for $\mathrm{Ni}$ [43], $\mathrm{Al}$ and $\mathrm{Cu}$ [1]. For stacking fault free energies even larger changes of between $-39 \%$ to $-86 \%$ were found from $0 \mathrm{~K}$ up to the melting point for the same three elements, $\mathrm{Ni}, \mathrm{Al}$, and $\mathrm{Cu}$ [12].

The fact that the overall impact of temperature on the surface free energy of TiN(001) is small should, however, not be misinterpreted as a small impact of explicit anharmonicity. The latter is in fact crucial in order to balance the diverging behavior obtained within the QHA as revealed by any of the green lines in Fig. 6. (These lines are for the MTP, but the DFT results show the same behavior.) The failure of the QHA can be traced back to the pronounced softening of interatomic forces in $\mathrm{TiN}$ at larger lattice constants, leading to the dynamical instability that has been discussed in the previous subsection. The dynamical instability and softening is observed primarily for the bulk as the $0 \mathrm{~K}$ surface relaxation stiffens the forces in the slab calculations. Fully anharmonic calculations are indispensable to get a sensible result for the surface free energy.

The shortcoming of the QHA is also reflected in the extreme sensitivity of the results to the choice of the atomic positions around which the dynamical matrix is calculated for the slab. The three green lines in Fig. 6 reflect the following choices:

(1) dashed green line (rel): relaxed atomic positions obtained at a lateral lattice constant $a=a_{\text {eq }}(T)$ fixed to the bulk equilibrium lattice constant $a_{\mathrm{eq}}(T)$ at the given temperature $T$;

(2) solid green line (resc): rescaled atomic positions obtained by rescaling the atomic positions with the 2 layers closest to the surface relaxed at the $0 \mathrm{~K}$ bulk equilibrium lattice constant $a_{\mathrm{eq}}(0 \mathrm{~K})$ with the factor $a_{\mathrm{eq}}(T) / a_{\mathrm{eq}}(0 \mathrm{~K})$;

(3) dotted green line (mean): mean atomic positions obtained from the AIMD at the corresponding temperature and lattice constant (cf. black circles in Fig. 7 below).

Regardless of which of these options is employed as the origin for the Taylor expansion of the potential energy surface, sooner or later a diverging behavior is found for the second order term (i.e., the dynamical matrix) as a function of temperature. We note that yet another choice of atomic positions for the expansion was proposed to be obtained by minimizing the free energy of the surface region [3]. Yet, whatever the choice, the QHA is a too severe approximation for the TiN(001) surface due to the dynamical instability at higher temperatures.

\section{Comparison of MTP and DFT results}

The MTP result for the surface free energy, obtained from the bulk-MTP and the slab-MTP, including the full impact 

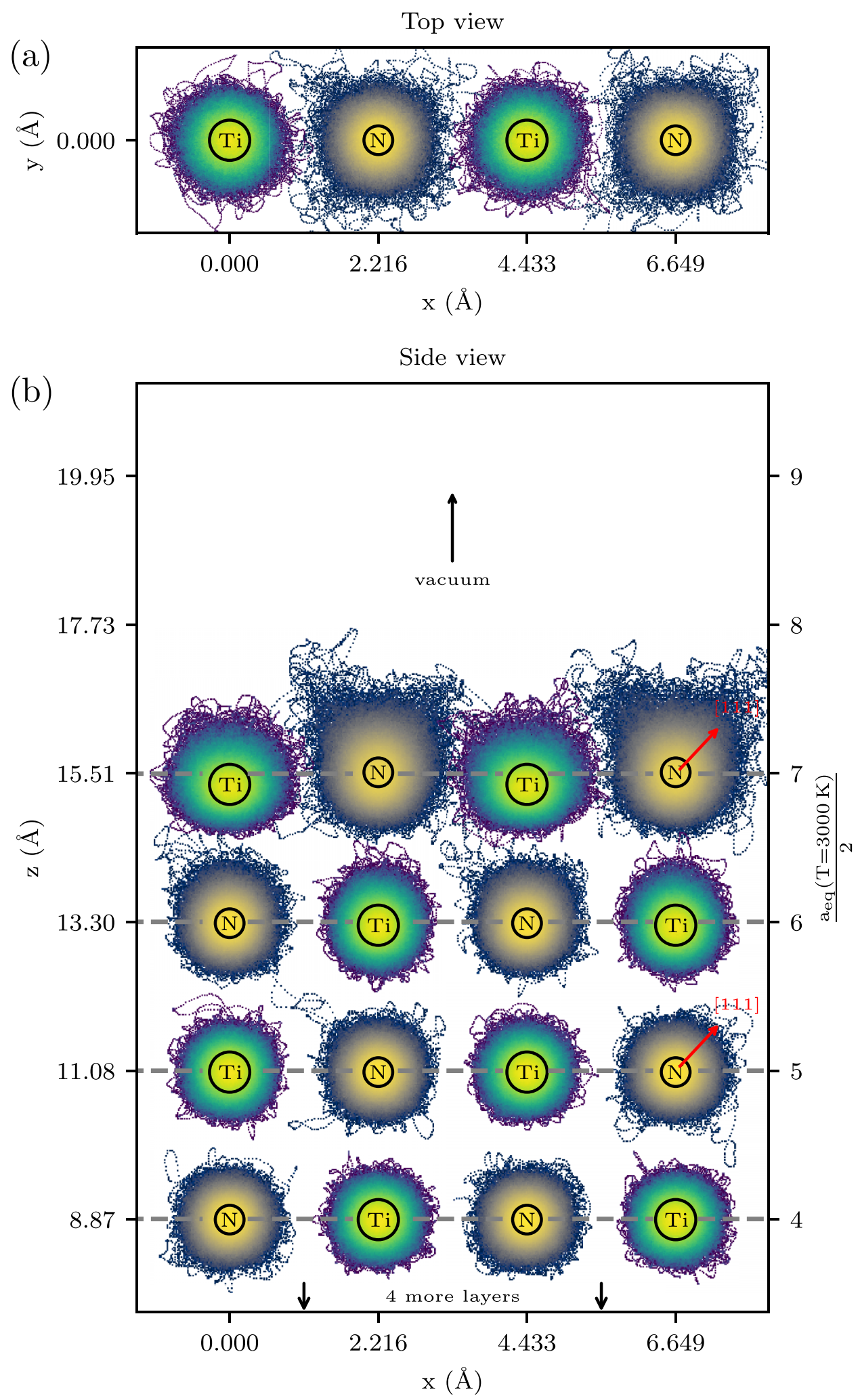

FIG. 7. Histogram of the trajectories from a slab-MTP MD of Ti and N atoms in TiN(001) at $3000 \mathrm{~K}$ : (a) Top view of the surface atoms and (b) side view of the first four layers. The atomic mean positions are indicated by the black circles. The circle sizes reflect the radii ratio between Ti and $\mathrm{N}$ [44].

of vibrations is shown in Fig. 6 by the orange dashed line. This result should be compared with the corresponding DFT result given by the solid orange line (part of which is hidden under the black solid line). The MTP curve is very close to the DFT curve over the full temperature window from $0 \mathrm{~K}$ to $3000 \mathrm{~K}$. The difference is at most a few meV $\AA^{2}$. The MTPs, fitted according to the prescription discussed in the Methodology section Sec. III, can thus be used to determine surface 
free energies with a high accuracy at small computational cost.

Interestingly, we do not only observe that the finite temperature, anharmonic properties are very well predicted by the MTPs. Moreover, the QHA results obtained from the MTPs are in good agreement with DFT (not shown in Fig. 6). The difference is somewhat larger than for the anharmonic results (several meV $\AA^{2}$ ) due to the sensitivity of the QHA as discussed in the previous subsection. Nevertheless, it is astonishing that the MTPs, which have been exclusively fitted to finite temperature DFT AIMD results, show a reasonable prediction also of the curvature of the potential energy surface at the $0 \mathrm{~K}$ equilibrium positions (i.e., the dynamical matrix).

\section{Atomic trajectories at high temperatures}

Figure 7 visualizes the slab-MTP MD trajectories of the TiN(001) slab at $3000 \mathrm{~K}$. In order to achieve reasonable statistics and to be able to analyze the shape of the atomic distributions, a very large number of MD steps is necessary. Figure 7 in particular is constructed from $100000 \mathrm{MD}$ steps with trajectories from symmetrically equivalent atoms mapped to every shown atom. Specifically, each shown atom imparts the information of 16 symmetrically equivalent atoms, i.e., each shown atom trajectory contains 1600000 steps. To reach such a number of steps directly with DFT AIMD would be computationally extremely demanding. The availability of the MTP that accurately reproduces the DFT anharmonic properties is therefore essential for an analysis as presented in the following.

For the surface $\mathrm{N}$ atoms (i.e., the $\mathrm{N}$ atoms in the first surface layer), we can clearly observe strongly anharmonic vibrations. The surface $\mathrm{N}$ atoms vibrate significantly further into the vacuum region than into the bulk. These pronounced vibrations into the vacuum are not only directed along the normal direction to the surface as one may have expected, but in particular along any of the symmetrically equivalent [111] directions (when extended from the bulk into the vacuum) and thus at an angle of $55.5^{\circ}$ with the surface. A careful inspection of the $\mathrm{N}$ atoms inside of the bulk reveals that already these bulk-like atoms have a preference to vibrate along the [111] directions. However, this preference becomes much more pronounced for the surface $\mathrm{N}$ atoms, particularly into the vacuum region.

Interestingly, despite the strongly anharmonic shape of the distribution of the surface $\mathrm{N}$ atoms, their mean positions (as extracted from the MD and indicated by the black circles in Fig. 7) are surprisingly close to the positions that would be expected for the bulk at this temperature. These expected mean positions from the bulk are indicated by the gray horizontal lines. The (vertical) distance between these lines corresponds to the nearest-neighbor distance from a bulk calculation at $3000 \mathrm{~K}$, i.e., including the effect of thermal expansion. We can see that the mean positions of the atoms inside of the bulk and also of the surface $\mathrm{N}$ atoms nicely fall on these gray lines. This means that on average the surface $\mathrm{N}$ atoms prefer the same distance to the lower lying $\mathrm{Ti}$ atoms as in the bulk.

For the surface $\mathrm{Ti}$ atoms, the situation is somewhat different. We can see that the mean positions are shifted into the bulk. This means that the nearest-neighbor distance to the lower lying $\mathrm{N}$ atoms is smaller than in the bulk at the respective temperature. Further, although not as pronounced as for the surface $\mathrm{N}$ atoms, one should appreciate that the surface $\mathrm{Ti}$ atoms exhibit anharmonic vibrations as well. These vibrations are now primarily directed along the surface normal, i.e., at an angle of $90^{\circ}$ with the surface. Interestingly, for the Ti atoms, it is not only the first surface layer that shows distinct vibrations from the bulk. A detailed analysis of the second layer of $\mathrm{Ti}$ atoms reveals that they vibrate with preference toward the $\mathrm{N}$ atoms in the first surface layer. This can be understood as a second-order effect induced by the strong anharmonic vibrations of these first-layer surface $\mathrm{N}$ atoms into the vacuum region.

\section{CONCLUSIONS}

We have developed an efficient methodology for accurately computing the fully anharmonic surface free energy from first principles by extending the TU-TILD method to surfaces. By using machine-learning MTPs, the computational effort of obtaining well converged results has been significantly reduced. The method has been applied to the industrially important TiN system, in particular to the $\mathrm{TiN}(001)$ surface.

As demonstrated, the quasiharmonic approximation breaks down due to a high-temperature dynamical instability that originates from the softening of the longitudinal acoustic mode at $q \approx 0.7 \frac{\pi}{a}[110]$. Inclusion of the fully anharmonic vibrations is crucial to obtain a stable thermal expansion coefficient and heat capacity, in excellent agreement with experimental data. Anharmonic effects are likewise critical to predict the temperature dependence of the surface free energy. Analysis of the high-temperature dynamic trajectories reveals symmetry-breaking vibrations of the surface atoms into the vacuum region. However, the mean positions of the $\mathrm{N}$ surface atoms remain close to their bulk positions at the respective temperature, and the mean positions of the Ti surface atoms even relax towards the bulk.

The full temperature effect on the $\mathrm{TiN}(001)$ surface free energy is comparably small, with the surface free energy changing from the $0 \mathrm{~K}$ value of around $78 \mathrm{meV}^{-2}$ to $73 \mathrm{meV}^{-2}$ at $3000 \mathrm{~K}$. This contrasts with previous results for $\mathrm{Al}(001)$ [3] and $\mathrm{TiO}_{2}(110)$ [4], which showed a stronger decrease of the surface energy with temperature. For TiN, the anharmonic vibrational contribution to the free energy in the bulk and at the (001) surface largely compensate each other in the surface free energy.

\section{ACKNOWLEDGMENTS}

This project was financially supported by the Swedish Foundation for Strategic Research via SSF Contract No. RMA15-0048 in the project CVD 2.0 and by the the European Research Council (ERC) under the EU's Horizon 2020 Research and Innovation Programme (Grant Agreement No. 865855). B.G. and A.V.S. acknowledge support from the collaborative DFG-RFBR Grant (Grants No. DFG KO 5080/3-1, No. DFG GR 3716/6-1, and No. RFBR 20-53-12012). B.G. acknowledges the support by the Stuttgart Center for Simulation Science (SimTech). The DFT simulations were 
performed on resources provided by the Swedish National Infrastructure for Computing (SNIC) at PDC (Stockholm) and NSC (Linköping) and through PRACE resources. We acknowledge PRACE for awarding us access to Piz Daint at CSCS, Switzerland. A.V.R. acknowledges a European Research Council grant, the VINNEX center Hero-m, financed by the Swedish Governmental Agency for Innovation Systems (VINNOVA), Swedish industry, and the Royal Institute of Technology (KTH). A.V.R. also gratefully acknowledges the financial support under the scope of the COMET program within the K2 Center Integrated Computational Material, Process and Product Engineering (IC-MPPE) (Project No. 859480). This program is supported by the Austrian Federal Ministries for Climate Action, Environment, Energy, Mobility, Innovation and Technology (BMK) and for Digital and Economic Affairs (BMDW), represented by the Austrian research funding association (FFG), and the federal states of Styria, Upper Austria, and Tyrol.
[1] A. Glensk, B. Grabowski, T. Hickel, and J. Neugebauer, Phys. Rev. X 4, 011018 (2014).

[2] X. Zhang, B. Grabowski, T. Hickel, and J. Neugebauer, Comput. Mater. Sci. 148, 249 (2018).

[3] U. Hansen, P. Vogl, and V. Fiorentini, Phys. Rev. B 60, 5055 (1999).

[4] H. Fox, A. P. Horsfield, and M. J. Gillan, J. Chem. Phys. 124, 134709 (2006).

[5] J. Xie, S. de Gironcoli, S. Baroni, and M. Scheffler, Phys. Rev. B 59, 970 (1999).

[6] S. Schönecker, X. Li, B. Johansson, S. K. Kwon, and L. Vitos, Sci. Rep. 5, 14860 (2015).

[7] J. Du, A. Zhang, Z. Guo, M. Yang, M. Li, F. Liu, and S. Xiong, Acta Mater. 161, 35 (2018).

[8] M. A. Gren, E. Fransson, and G. Wahnström, Int. J. Refract. Met. Hard Mater. 87, 105114 (2020).

[9] D. Scheiber, O. Renk, M. Popov, and L. Romaner, Phys. Rev. B 101, 174103 (2020).

[10] W. Tyson, Can. Metall. Q. 14, 307 (1975).

[11] A. I. Duff, T. Davey, D. Korbmacher, A. Glensk, B. Grabowski, J. Neugebauer, and M. W. Finnis, Phys. Rev. B 91, 214311 (2015).

[12] X. Zhang, B. Grabowski, F. Körmann, A. V. Ruban, Y. Gong, R. C. Reed, T. Hickel, and J. Neugebauer, Phys. Rev. B 98, 224106 (2018).

[13] B. Grabowski, Y. Ikeda, P. Srinivasan, F. Körmann, C. Freysoldt, A. I. Duff, A. Shapeev, and J. Neugebauer, npj Comput. Mater. 5, 80 (2019).

[14] H. E. Rebenne and D. G. Bhat, Surf. Coat. Technol. 63, 1 (1994).

[15] M. Marlo and V. Milman, Phys. Rev. B 62, 2899 (2000).

[16] A. Dunand, H. D. Flack, and K. Yvon, Phys. Rev. B 31, 2299 (1985).

[17] S. Dal Forno and J. Lischner, Phys. Rev. Materials 3, 115203 (2019).

[18] A. S. Bochkarev, M. N. Popov, V. I. Razumovskiy, J. Spitaler, and P. Puschnig, Phys. Rev. B 94, 104303 (2016).

[19] S. Iikubo, H. Ohtani, and M. Hasebe, Mater. Trans. 51, 574 (2010).

[20] A. Wang, S. Shang, D. Zhao, J. Wang, L. Chen, Y. Du, Z.-K. Liu, T. Xu, and S. Wang, Calphad 37, 126 (2012).

[21] M. Bartosik, D. Holec, D. Apel, M. Klaus, C. Genzel, J. Keckes, M. Arndt, P. Polcik, C. M. Koller, and P. H. Mayrhofer, Scr. Mater. 127, 182 (2017).
[22] P. Steneteg, O. Hellman, O. Vekilova, N. Shulumba, F. Tasnádi, and I. Abrikosov, Phys. Rev. B 87, 094114 (2013).

[23] A. V. Shapeev, Multiscale Model. Simul. 14, 1153 (2016).

[24] I. S. Novikov, K. Gubaev, E. V. Podryabinkin, and A. V. Shapeev, Machine Learning: Science and Technology 2, 025002 (2021).

[25] D. Korbmacher, Ab initio study of strongly anharmonic and dynamically unstable systems, Ph.D. thesis, Ruhr-Universität Bochum, 2019.

[26] P. E. Blöchl, Phys. Rev. B 50, 17953 (1994).

[27] G. Kresse and J. Hafner, Phys. Rev. B 47, 558 (1993) 49, 14251 (1994). G. Kresse and J. Furthmüller, ibid. 54, 11169 (1996). Comput. Mater. Sci. 6, 15 (1996)..

[28] G. Kresse and D. Joubert, Phys. Rev. B 59, 1758 (1999).

[29] J. P. Perdew, K. Burke, and M. Ernzerhof, Phys. Rev. Lett. 77, 3865 (1996).

[30] M. Methfessel and A. T. Paxton, Phys. Rev. B 40, 3616 (1989).

[31] P. Vinet, J. H. Rose, J. Ferrante, and J. R. Smith, J. Phys.: Condens. Matter 1, 1941 (1989).

[32] X. Zhang, B. Grabowski, F. Körmann, C. Freysoldt, and J. Neugebauer, Phys. Rev. B 95, 165126 (2017).

[33] N. D. Mermin, Phys. Rev. 137, A1441 (1965).

[34] B. Grabowski, P. Söderlind, T. Hickel, and J. Neugebauer, Phys. Rev. B 84, 214107 (2011).

[35] H. J. Monkhorst and J. D. Pack, Phys. Rev. B 13, 5188 (1976).

[36] B. Grabowski, L. Ismer, T. Hickel, and J. Neugebauer, Phys. Rev. B 79, 134106 (2009).

[37] W. van Gunsteren and H. Berendsen, Mol. Phys. 45, 637 (1982).

[38] A. Togo and I. Tanaka, Scr. Mater. 108, 1 (2015).

[39] L. Toth, Transition Metal Carbides and Nitrides (Academic Press, New York, 1971).

[40] J. P. Perdew, A. Ruzsinszky, G. I. Csonka, O. A. Vydrov, G. E. Scuseria, L. A. Constantin, X. Zhou, and K. Burke, Phys. Rev. Lett. 100, 136406 (2008).

[41] M. W. Chase Jr., J. Phys. Chem. Ref. Data, Monograph 9, 1612 (1998).

[42] W. Lengauer, S. Binder, K. Aigner, P. Ettmayer, A. Guillou, J. Debuigne, and G. Groboth, J. Alloys Compd. 217, 137 (1995).

[43] Y. Gong, B. Grabowski, A. Glensk, F. Körmann, J. Neugebauer, and R. C. Reed, Phys. Rev. B 97, 214106 (2018).

[44] A. Forslund and A. Ruban, Comput. Mater. Sci. 183, 109813 (2020). 